\title{
Ear, nose and throat manifestations of Lyme disease
}

\author{
T.H. J. Lesser F.R.C.S., J. C. Dort M.D. F.R.C.S.C., D. P. B. Simmen M.D. (Zurich, Switzerland)
}

\begin{abstract}
The manifestations of Lyme disease as they may present to the ENT surgeon are discussed. The most important ENT symptom is facial palsy. Particularly when combined with other cranial palsies, systemic illness or signs of meningeal irritation, the diagnosis must be considered. Three case reports are used to illustrate the presentation and diagnosis and treatment of Lyme disease. The characteristics of the disease are reviewed and the limitations of serological testing outlined. The literature has concentrated on bilateral or relapsing facial palsy. A review of palsies in Zurich that presented to the ENT clinic found only unilateral and partial palsies. The diagnosis should be considered in every case of facial palsy of unknown aetiology especially in children.
\end{abstract}

\section{Introduction}

The purpose of this paper is to bring to the attention of the practicing ENT surgeon the manifestations of Lyme disease as they may present in the clinic. The symptom of most importance is facial palsy and this will be given most attention. The otological symptoms of tinnitus, deafness and dizziness are less common but are also important as they, along with the general symptoms, may alert the clinician to the possibility of this disease. The presentation, problems in diagnosis and the treatment will be illustrated with three typical cases presenting in the last few months to the ENT department in Zurich. Lyme disease is a multi-system tick borne spirochetal infection first described in 1975 in Lyme, Connecticut. The historical aspects of the definition of the disease are well documented by Habicht et al., (1987). Since then, the spirochaete has been named Borrelia burgdorferi and the major vectors discovered to be Ixodes dammini and pacificus in the U.S.A. and Ixodes ricinus in mainland Europe and Great Britain.

The clinical picture starts occasionally with a history of a tick bite whilst walking or riding in a wooded area or moorland. A week to a month later systemic 'flu like' symptoms may occur and a distinctive skin lesion 'erythema chronicum migrans' appears. This begins as a red macule or papule and expands to become an annular lesion which may be many centimetres in diameter. The symptoms that accompany the rash may be mild or very severe and include fever, malaise, neck stiffness, headache, depression, myalgia, arthralgia and of note to the ENT surgeon, pharyngitis.

The rash itself will last for around three weeks. Days to months after the commencement of the rash, neurological and myocardial conduction abnormalities may occur. Arthritis, similar in character to rheumatoid arthritis, occurs as the late stage of the disease. The arthritis, like the cardiac abnormalities, occur especially in the USA. They are not so prominent a feature in
Europe where the neurological manifestations predominate. (Muhlemann and Wright, 1987). Serological testing for IgM and IgG titres is available but often is not of diagnostic help both because of lack of specificity and also as serial testing is usually required. The diagnosis therefore remains clinical.

\section{Case reports}

Case 1

A 50-year-old woman was referred to the clinic as a case of Bell's palsy in July. She gave a history of weakness on the left side of her face for one day. She had felt unwell for eight days with headache and generalized aches and pains. She had noticed ringing noises in the left ear. There was no history of tick bite or rash but she was a keen gardener and took her dog on daily walks in the woods. On examination she had a left lower motor neuron partial facial palsy and no other abnormalities. Electroneuronography showed 66 per cent degeneration. Audiometry was normal. On blood testing, the ESR was normal and Lyme titre borderline. After three days, the facial palsy was showing some improvement. One week after the onset the face was almost normal but the general symptoms had worsened with headache and pains in the arms and neck particularly at night. She was admitted and found to have no new signs but was considered to have minimal meningism. The cerebrospinal fluid was clear and titres for Borrelia burgdorferi were borderline. A diagnosis of Lyme disease was made on the clinical grounds of headache, meningeal symptoms and facial palsy. She was treated with Penicillin G and was home and walking her dog again after one week.

\section{Case 2}

A 31-year-old Swiss female presented in July with a four week history of malaise, sore throat, earache and 
tinnitus on the left side. Three days prior to presentation, she noticed her face was weak and she felt feverish with a headache. There was no definitive history of tick bite but she was a frequent hiker. On examination, she had a lower motor neuron partial left facial palsy, a left sided $30 \mathrm{~dB}$ sensorineural hearing loss, and a left glossopharyngeal nerve palsy. The general examination revealed no other abnormality. In particular, there was no evidence of arthritis or meningism, the other cranial nerves were normal. Electroneuronography showed a 40 per cent degeneration. An electrocardiogram showed no conduction abnormality. As there was no dysequilibrium or nystagmus, caloric tests were not performed. A clinical diagnosis of Lyme disease was made and she was treated with Vibramycin. Four days later the systemic symptoms had gone completely and the facial palsy had returned to normal. The sensorineural deafness had returned to normal in the low tones but there was some residual loss at $6,000,8,000$ and $12,000 \mathrm{~Hz}$ on the left. Serological testing showed a Borrelia burgdorferi titre of 80 at presentation and 80 three months later. This is considered to represent infection in the past but not diagnostic of infection in the current episode.

\section{Case 3}

A 30-month-old Canadian male was visiting Switzerland with his parents. Several days after hiking in the woods, the patients mother observed a tick attached to the canal surface of the right tragus. The tick was removed and the patient was well until three weeks later. At that time an erythematous, macular rash appeared on the right cheek. The skin rash had a clearly demarcated border and it was noted to change in appearance from day to day. There were no other symptoms until two weeks later (five weeks post tick bite) when the patient developed a low-grade fever $\left(38.5^{\circ} \mathrm{C}\right)$ and lassitude. The rash had now enlarged to $4 \times 6 \mathrm{~cm}$ and an area of clearing was evident in the middle of lesion. (Fig. 1).

General physical examination was normal except for the skin rash and low grade fever. There was no evidence of meningitis, arthritis or cervical adenopathy. The pinna was normal and there was no sign of local infection of the previous tick bite. Facial movements were normal.

A diagnosis of Lyme disease was made based on the history of a tick bite and the characteristic rash (Erythema chronicum migrans). The child was placed on a prophylactic course of erythromycin (because of penicillin allergy) and 48 hours later the skin rash, fever and malaise had resolved. There has been no recurrence of symptoms since completing the antibiotics.

\section{Discussion}

This disease has existed and been recognized for nearly a century. In 1909, Arvid Afzelius a swedish physician is reported to have described an expanding red skin rash in patients bitten by the tick Ixodes ricinus. (Habicht et al., 1987). Since its recognition in Lyme, Connecticut, the reported incidence has increased from 59 in 1975 to 863 cases in 1985 in the USA. In the UK in 1986, 68 cases were recorded by Muhlemann and Wright (1987) who also identified two areas in which the infec- tion was harboured in the deer population, the New Forest and Thetford. It is likely to be present in many different areas as migratory birds can act as distant carriers of the spirochaete. The patients themselves will carry the disease back from holidays, the main candidates being those on walking holidays in the scenic parts of the middle of western Europe and the USA. As awareness of this disease increases the case numbers will also increase. Zurich and the surrounding countryside form one of the many European endemic areas.

The diagnosis has to be made on clinical grounds although many aspects of the history are inconsistant. The case histories presented are typical in that the tick was noticed in only one. The history of a tick bite or skin rash is helpful when present but it is absent in half to twothirds of patients with the neurological stage of the disease (Hanny and Hauselmann, 1987). The case histories also demonstrate how antibody testing is not reliable when negative. Furthermore a positive titre is not necessarily specific. In northern Switzerland, Satz et al. (1988) randomly tested 241 persons for serum IgG titre to Borrelia burgdorferi. Twenty-three ( 9.5 per cent) had a raised titre and only two ( 0.8 per cent $)$ had any history of infection. The antibody test also cross reacts with other spirochaetes and a simultaneous VDRL or other test for syphilis needs to be performed. A further problem noted by Hanny and Hauselmann (1987) is that the severity of the disease is not related to the height of the titre when positive. The IgM titre may be only raised for a few days after the infection and may mislead if it is not raised. The IgG titre may not rise to a diagnostic level for weeks and then can only be used to confirm the diagnosis in retrospect.

Other laboratory tests can also be misleading; for instance, the ESR is only raised in half the patients with neurological manifestations.

The facial nerve palsy is the symptom of most note to the ENT surgeon. It is the most common neurological sign and can often be the first specific lesion in the disease. This is illustrated by Case 1 . In neurological series, it is reported as occurring 49 per cent in Switzerland, 64 per cent in West Germany, 41 per cent in Sweden and 50 per cent in the USA; of these 20 per cent were bilateral palsies. (Hanny and Hauselmann, 1987). These patients all had other systemic problems and are not necessarily the ones who present primarily to the ENT surgeon. To see if those that present to the ENT clinic have the same profile we looked at the charts of the patients presenting in the summer months of 1986 and 1987 to the outpatients in Zurich. The summer months were chosen as this is the time when the ticks are active. Of 23 patients presenting with facial palsy between May and November, 11 had positive or rising titres, all had lower motor neuron palsies which were partial in nature, none had bilateral palsy. Indeed of the last 18 patients who presented to the clinic in Zurich with bilateral palsies (either simultaneously or concurrently), none had a diagnosis of Lyme disease.

The prognosis of the facial palsy is excellent, even in those patients with other neurological and often permanent problems. Of 20 palsies in the neurological series from Zurich only one had 100 per cent degeneration (Hanny and Hauselmann, 1987). Of the 11 Lyme patients presenting through the ENT outpatients the 


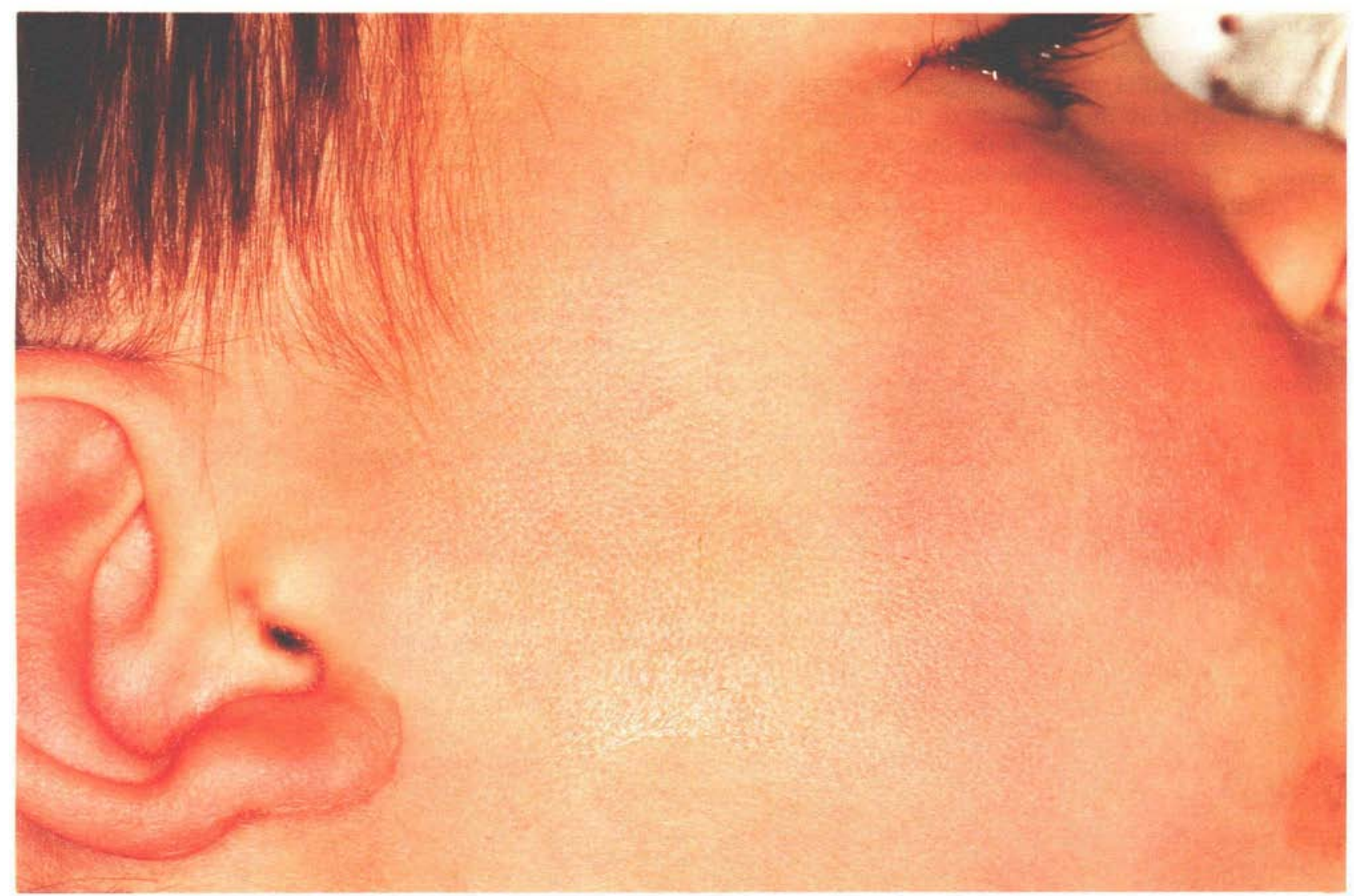

FIG. 1

Child's face with Erythema chronicum migrans. Case 3.

greatest degeneration was 69 per cent and all recovered completely within a few weeks.

Reik et al. (1979) found in a neurological series, seven out of 18 patients had a VIIth nerve palsy but only two had this as an isolated finding. Residual weakness after treatment occurred. They stated the now accepted finding that the lower motor palsy may be bilateral or unilateral and may be relapsing.

In the largest series published, Clark et al. (1985) from the USA found 23 per cent of their 101 patients had a bilateral palsy. This again represents patients with all variations of the disease and does not relate to patients who are seen de novo in the ENT clinic, although it must be borne in mind that it may point to a further difference between the USA and European disease.

In endemic areas Lyme disease is the most common cause of childhood facial palsies. (Christen et al., 1989).

In adults the most common cause remains 'Bells palsy' though as this is a diagnosis of exclusion, if there is any possibility of a visit to an endemic area, or any sign or symptom other than the palsy the diagnosis of Lyme disease should be considered. The ENT symptoms also include palsies involving the cranial nerves. As well as the glossopharyngeal nerve palsy reported here it may also affect the recurrent laryngeal nerve (Schroeter $e t$ al., 1988).

Otological symptoms are less common and appear to be part of the more severe disease. Dizziness affects 12 per cent of patients overall, this may be a vestibular neuronitis or even a cerebellar ataxia may occur. Decreased hearing and tinnitus may also involve the otolaryngol- ogist, though the exact incidence of these symptoms is not usually recorded. Diehl and Holtmann (1989) report four sudden hearing losses, three with tinnitus and one vestibular neuronitis in a group of $15 \mathrm{ENT}$ patients with Lyme disease. It is interesting that the patient in case 2 had recovery of hearing following treatment. The hearing loss is usually recorded as total and recovery is not apparent. Tinnitus may also remain despite treatment. The patient in case 2 also represents the cranial neuropathies well and shows how they can respond to oral antibiotics. Though there have been doubts raised as to whether treatment has any effect on the natural history of the neurological symptoms of the disease. Case 2 also demonstrates how the diagnosis must be made on clinical grounds and how relying on blood serology can be misleading.

Treatment is with penicillin, erythromycin or tetracycline. There have been occasional resistance reported and early trials of cephalosporin in the severity effected neurological patients have shown promising results. Currently all patients in Zurich with a facial palsy of unknown aetiology have Lyme titres taken. If positive, oral antibiotics are given. Patients with systemic symptoms are admitted for intravenous therapy. The total course is three weeks.

\section{Conclusion}

The ENT surgeon should be aware that facial palsy may be due to Lyme disease. This is particularly so in those presenting in summer and autumn. A history of 
hiking, tick bites and rash should be sought. Though they are only noted by less than half the patients. The systemic symptoms and neurological signs and symptoms should alert the clinician to the diagnosis. The emphasis on bilateral palsy in the literature may be misleading. The diagnosis of this disease is primarily clinical. Antibody titres can be of help but cannot be relied on.

\section{References}

Christen, H. J., Bartian, N., Hanefeld, F., Thomssen, R. (1989) Lyme borreliosis. Main cause of acute peripheral facial palsy in childhood. Monatsschrift Kinderheilkdindue, 137: 151-157.

Clark, J. R., Carlson, R. D., Sasaki, C. T., Pachner, A. R., Steer, A. C. Facial paralysis in Lyme disease. Laryngoscope, 95: 1341-1345.

Diehl, G. E., Holtmann, S. (1989) Die Lyme-Borreliose und ihre Bedeutung fur den HNO-Arzt. Laryngologie-Rhonologie-Otologie, 68: 81-89.
Harbicht, G. S., Beck, G., Benach, J. L. (1987) Lyme disease. Scientific American, 267: 60-65.

Hanny, P. E., Hauselmann, H. J. (1987) Die Lyme-krankheit aus de sicht des neurologen. Schweizerische medizinische Wochenschrift, 117: 901-915.

Muhlemann, M. F., Wright, D. J. M. (1987) Emerging pattern of Lyme disease in the United Kingdom and Irish Republic. Lancet, 260-262.

Reik, L., Steere, A. C., Bartenhagn, N. H., Shope, R. E., Malawista, S. E. (1979) Neurologic abnormalities of Lyme disease. Medicine, 58: 281-294.

Satz, N., Ackermann, R., Gern L., Aeschlimann, A., Ott, A. Knoblauch, M. (1988) Zur epidemiologie der infection mit Borrelia Burgerdorferi. Schweizerische Medizimiche Wochenshrift, 118: $422-426$.

Schroeter, V., Belz; G. G., Blenk, H. (1988). Paralysis of recurrent laryngeal nerve in Lyme disease. Lancet, 8622 p1245.

Address for correspondence:

T. H. J. Lesser,

Department of ENT,

University Hospital of Wales,

Cardiff, Wales, U.K. 